Pacific Journal of Mathematics

THE PERTURBATION THEORY FOR LINEAR OPERATORS OF
DISCRETE TYPE 


\section{THE PERTURBATION THEORY FOR LINEAR OPERATORS OF DISCRETE TYPE}

\section{BINGREN}

Using the theory of unconditional bases, we discuss the perturbation theory of linear operators of discrete type.

The principal abstract perturbation theorem about discrete spectral operators was introduced by J. T. Schwartz, and extended by H. P. Kramer to the general case ([1], XIX.2 Theorem 7). In this paper, we shall give a simple proof for Schwartz-Kramer's Theorem by using the theory of unconditional bases, and omit the condition of weak completeness in their theorem. In the proof of [1], XIX.2 Theorem 7, because of using [1], XVIII.2 Corollary 33, so that it needs the condition of weak completeness. On the other hand, all perturbant generalized eigenvectors consist of an unconditional basis, so we can prove the theorem without using the above corollary and omit the condition of weak completeness.

Definition 1. A linear operator $T$ in Banach space $B$ is called discrete type $((D)$ type), if $\rho(T) \neq \varnothing$, and there exist an unconditional basis $\left\{x_{n}\right\}$ of $B$, a sequence of complex numbers $\left\{\lambda_{n}\right\}$ and a positive integer $N$, such that $\lim _{n}\left|\lambda_{n}\right|=+\infty, \lambda_{n} \neq \lambda_{m}, \forall n, m \in \mathbf{N}, m>N$ and $n \neq m, \quad T x_{n}=\lambda_{n} x_{n}, \quad \forall n>N, T\left[x_{1}, \ldots, x_{N}\right] \subset\left[x_{1}, \ldots, x_{N}\right]$ and $\sigma\left(T \mid\left[x_{1}, \ldots, x_{N}\right]\right)=\left\{\lambda_{1}, \ldots, \lambda_{N}\right\}$.

PROPOSITION 2. Let $T$ be a linear operator of $(D)$ type in Banach space $B,\left\{x_{n}\right\},\left\{\lambda_{n}\right\}$ and $N$ as in Definition 1. Then $\sigma(T)=\left\{\lambda_{n}\right\}$,

$$
\begin{aligned}
\mathscr{D}(T) & =\left\{x \in B \mid \text { if } x=\sum_{n} \alpha_{n} x_{n}, \text { then } \sum_{n>N} \lambda_{n} \alpha_{n} x_{n} \in B\right\} \\
T x & =\sum_{n=1}^{N} \alpha_{n} T x_{n}+\sum_{n>N} \lambda_{n} \alpha_{n} x_{n}, \quad \forall x=\sum_{n} \alpha_{n} x_{n} \in \mathscr{D}(T) .
\end{aligned}
$$

However, for each $\lambda \notin \sigma(T), R(\lambda, T)=(T-\lambda I)^{-1}$ is compact and

$$
\begin{aligned}
R(\lambda, T) x & =\sum_{n=1}^{N} \alpha_{n}(T-\lambda I)^{-1} x_{n}+\sum_{n>N} \frac{\alpha_{n}}{\lambda_{n}-\lambda} x_{n}, \\
\forall x & =\sum_{n} \alpha_{n} x_{n} \in B .
\end{aligned}
$$


Proof. Define a linear operator $T_{0}$ in $B$ as follows

$$
\begin{aligned}
\mathcal{Q}\left(T_{0}\right) & =\left\{x \in B \mid \text { if } x=\sum_{n} \alpha_{n} x_{n}, \text { then } \sum_{n>N} \lambda_{n} \alpha_{n} x_{n} \in B\right\}, \\
T_{0} x & =\sum_{n=1}^{N} \alpha_{n} T x_{n}+\sum_{n>N} \lambda_{n} \alpha_{n} x_{n}, \quad \forall x=\sum_{n} \alpha_{n} x_{n} \in \mathcal{D}\left(T_{0}\right) .
\end{aligned}
$$

Because $T$ is closed, so $T \supset T_{0}$. Without loss of generality, we can assume $0 \in \rho(T)$. Then by $\left|\lambda_{n}\right| \rightarrow \infty$ and [3], Ch. II Lemma 16.1, let

$$
y=\sum_{n=1}^{N} \alpha_{n} T^{-1} x_{n}+\sum_{n>N} \frac{\alpha_{n}}{\lambda_{n}} x_{n} \in \mathscr{D}\left(T_{0}\right)
$$

for $x=\Sigma_{n} \alpha_{n} x_{n} \in B$, and $T_{0} y=x$, so that $T_{0} \mathscr{D}\left(T_{0}\right)=B$. Therefore $T=$ $T_{0}$.

If $\lambda \neq \lambda_{n}, \forall n$, because of $\left|\lambda-\lambda_{n}\right| \rightarrow \infty$ and above Lemma 16.1, it is easy to see $(T-\lambda I) \mathscr{D}(T)=B$. So that $\sigma(T)=\left\{\lambda_{n}\right\}$, and we have the formula about $R(\lambda, T)$.

We can assume $\left\|x_{n}\right\|=1, \forall n$. Let $f_{m} \in B^{*}$, such that $f_{m}\left(x_{n}\right)=\delta_{n, m}$, $\forall n, m$. Then there exists a positive constant $M_{1}$, such that $\left\|f_{m}\right\| \leq M_{1}$, $\forall m$.

For each $n$, let $P_{n}, Q_{n}$ be the projections, such that $P_{n}+Q_{n}=I$, and $P_{n} B=\left[x_{1}, \ldots, x_{n}\right], Q_{n} B=\left[x_{n+1}, \ldots, x_{m}, \ldots\right]$. By [3], Ch. II Th. 17.1, there exists a positive constant $M_{2}$, such that $\left\|Q_{n}\right\| \leq M_{2}, \forall n$.

Again by above Th. 17.1, there exists a positive constant $M_{3}$, such that

$$
\left\|\sum_{n} \beta_{n} \alpha_{n} x_{n}\right\| \leq M_{3}\|x\|, \quad \forall x=\sum_{n} \alpha_{n} x_{n} \in B \text { and }\left|\beta_{n}\right| \leq 1(\forall n) .
$$

Let $\lambda \in \rho(T)$ and $\left\{y_{n}\right\}$ be a bounded sequence of $B$, i.e. $\left\|y_{n}\right\| \leq M_{4}$, $\forall n$. Because $\left|f_{m}\left(y_{n}\right)\right| \leq M_{1} M_{4}$, we can assume that

$$
f_{m}\left(y_{n}\right)=: \alpha_{m}^{(n)} \rightarrow \alpha_{m}, \quad \forall m
$$

(replacing a subsequence of $\left\{y_{n}\right\}$, if necessary). For $\varepsilon>0$, there exists $N_{1}(>N)$ such that $\left|1 /\left(\lambda_{n}-\lambda\right)\right|<\varepsilon, \forall n \geq N_{1}$. Then for sufficiently large $n, m$

$$
\begin{array}{r}
\left\|R(\lambda, T) Q_{N}\left(y_{n}-y_{m}\right)\right\| \leq \sum_{k=N+1}^{N_{1}}\left|\frac{\alpha_{k}^{(n)}-\alpha_{k}^{(m)}}{\lambda_{k}-\lambda}\right|+\left\|\sum_{k>N_{1}} \frac{\alpha_{k}^{(n)}-\alpha_{k}^{(m)}}{\lambda_{k}-\lambda} x_{k}\right\| \\
\leq \varepsilon+\varepsilon M_{3}\left\|Q_{N_{1}} y_{n}-Q_{N_{1}} y_{m}\right\| \leq\left(1+2 M_{2} M_{3} M_{4}\right) \varepsilon
\end{array}
$$

Therefore $R(\lambda, T) Q_{N}$ and $R(\lambda, T)$ are compact. 
LEMMA 3. Let $\left\{x_{n}\right\}$ be an unconditional basis of Banach space $B, J$ be $a$ subset of $\mathbf{N}$. Then $\left\{x_{n} \mid n \in J\right\}$ is an unconditional basis of $\left[x_{n} \mid n \in J\right]$, where $\left[x_{n} \mid n \in J\right]$ is the closed subspace generated by $\left\{x_{n} \mid n \in J\right\}$, and

$$
B=\left[x_{n} \mid n \in J\right]+\left[x_{n} \mid n \notin J\right] .
$$

However, let $P(J)$ be the projection from $B$ onto $\left[x_{n} \mid n \in J\right]$ such that $(I-P(J)) B=\left[x_{n} \mid n \notin J\right]$, then $J \rightarrow P(J)$ is countably additive in the strong operator topology from the $\sigma$-field of all subsets of $\mathbf{N}$ into the projections in $B$, and $P(\mathbf{N})=I, P(\varnothing)=0$.

Proof. Let $P_{n}$ be the projection from $B$ to $\left[x_{n}\right]$ such that $\left(I-P_{n}\right) B=$ $\left[x_{m} \mid m \neq n\right], \forall n$. If $x=\Sigma_{n} \alpha_{n} x_{n} \in\left[x_{n} \mid n \in J\right]$, by $P_{m} x=0, \forall m \notin J$, so that $\alpha_{m}=0, \forall m \notin J$ and $x=\sum_{n \in J} \alpha_{n} x_{n}$. This series is also unconditionally convergent, therefore $\left\{x_{n} \mid n \in J\right\}$ is an unconditional basis of $\left[x_{n} \mid n \in J\right]$. Similarly, $\left\{x_{n} \mid n \notin J\right\}$ is an unconditional basis of $\left[x_{n} \mid n \notin J\right]$, so that

$$
B=\left[x_{n} \mid n \in J\right] \dot{+}\left[x_{n} \mid n \notin J\right] .
$$

We notice the following fact: if $x=\Sigma_{n} \alpha_{n} x_{n} \in B$ and $\varepsilon>0$, then there exists a positive integer $N$, such that

$$
\left\|\sum_{n \in \Lambda} \alpha_{n} x_{n}\right\|<\varepsilon
$$

where $\Lambda$ is an arbitrary subset of $N$ and $\Lambda \cap\{1, \ldots, N\}=\varnothing$. In fact, we have $N$ such that

$$
\left\|\sum_{n>N} \alpha_{n} x_{n}\right\|<\frac{\varepsilon}{M}
$$

where $M$ is the constant such that $\left\|\Sigma_{n} \varepsilon_{n} \beta_{n} x_{n}\right\| \leq M\|y\|, \forall y=\Sigma_{n} \beta_{n} x_{n} \in$ $B$ and $\left|\varepsilon_{n}\right| \leq 1, \forall n \in \mathbf{N}$ ([3], Ch. II, Th. 17.1). Let

$$
\varepsilon_{n}= \begin{cases}1 & n \in \Lambda \\ 0 & \text { otherwise }\end{cases}
$$

then

$$
\left\|\sum_{n \in \Lambda} \alpha_{n} x_{n}\right\|=\left\|\sum_{n>N} \varepsilon_{n} \alpha_{n} x_{n}\right\| \leq M\left\|\sum_{n>N} \alpha_{n} x_{n}\right\|<\varepsilon .
$$

Now let $J_{l} \subset \mathbf{N}, J_{l} \cap J_{j}=\varnothing, \forall i \neq j, J=\cup_{i=1}^{\infty} J_{i}$, and $x=\Sigma_{n} \alpha_{n} x_{n} \in$ $B, \varepsilon>0$. Take above $N$ and a positive integer $K$ such that

$$
\bigcup_{i=1}^{K} J_{l} \supset J \cap\{1, \ldots, N\}
$$


then

$$
\left\|\left(\sum_{i=1}^{K} P\left(J_{l}\right)-P(J)\right) x\right\|=\left\|\sum_{n \in \Lambda} \alpha_{n} x_{n}\right\|<\varepsilon
$$

where $\Lambda=J \backslash \cup_{t=1}^{K} J_{t}$ and $\Lambda \cap\{1, \ldots, N\}=\varnothing$. So that

$$
P(J)=\mathrm{s}-\lim _{K} \sum_{i=1}^{K} P\left(J_{l}\right)
$$

and $J \rightarrow P(J)$ is countably additive in the strong operator topology.

Now we recall that a linear operator $T$ in Banach space $B$ is spectral as in [1], XVIII. 2, Definition 1, and $T$ is discrete as in [1], XIX. 2, Definition 1, i.e. every resolvent $R(\lambda, T)$ of $T$ is compact. We also say that a discrete spectral operator $T$ satisfies condition $(F)$, if for all but a finite number of spectral points $\lambda$, the space of generalized eigenvectors of $T$ corresponding to $\lambda$ is one-dimensional.

Proposition 4. Let $T$ be a linear operator in Banach space B. Then $T$ is $(D)$ type, if and only if, $T$ is a discrete spectral operator which satisfies condition $(F)$.

Proof. Let $T$ be $(D)$ type, $\left\{x_{n}\right\},\left\{\lambda_{n}\right\}$ and $N$ as in Definition 1. We assume that $\left\{\lambda_{1}, \ldots, \lambda_{N}\right\}=\left\{\lambda_{1}, \ldots, \lambda_{k}\right\}$, where $k \leq N$ and $\lambda_{i} \neq \lambda_{j}, \forall 1 \leq$ $i \neq j \leq k$. If $B_{i}$ is the space of generalized eigenvectors of $T$ corresponding to $\lambda_{\imath}, 1 \leq i \leq k$, then $N=\sum_{l=1}^{k} \operatorname{dim} B_{i}$. We can also assume that there is a partition $\{1, \ldots, N\}=\cup_{i=1}^{k} \Lambda_{l}$, such that $B_{i}=\left[x_{n} \mid n \in \Lambda_{i}\right], 1 \leq i \leq k$. Let $B_{n}=\left[x_{n}\right], \forall n>N$ and $\mathscr{G}$ be all Borel subsets of complex plane $\mathbf{C}$, and

$$
P(\Delta)=\dot{+}\left\{B_{n} \mid \lambda_{n} \in \Delta\right\} \quad \forall \Delta \in \Re
$$

then by Lemma $3, \Delta \rightarrow P(\Delta)$ is countably additive in the strong operator topology.

Let $\Delta \in \mathscr{B}$ and $x \in \mathscr{D}(T) \cap P(\Delta) B$, we can write

$$
x=\sum_{\substack{n \in \Lambda_{l} \\ \text { and } \lambda_{t} \in \Delta}} \alpha_{n} x_{n}+\sum_{\substack{n>N \\ \text { and } \lambda_{l} \in \Delta}} \alpha_{n} x_{n}
$$

by Proposition 2,

$$
T x=\sum_{\substack{n \in \Lambda_{1} \\ \text { and } \lambda_{1} \in \Delta}} \alpha_{n} T x_{n}+\sum_{\substack{n>N_{1} \\ \text { and } \lambda_{n} \in \Delta}} \lambda_{n} \alpha_{n} x_{n} \in P(\Delta) B .
$$


However, $T \mid P(\Delta) B$ also satisfies the assumptions of Proposition 2, so that $\sigma(T \mid P(\Delta) B)=\left\{\lambda_{l} \mid \lambda_{i} \in \Delta\right\} \subset \bar{\Delta}$. Therefore $T$ is a discrete spectral operator which satisfies condition $(F)$.

Conversely, let $T$ be a discrete spectral operator which satisfies condition $(F)$. Let $P(\cdot)$ be the resolution of the identity for $T$ and assume that the different eigenvalues of $T$ are $\lambda_{1}, \ldots, \lambda_{k}, \lambda_{N+1}, \ldots, \lambda_{n}, \ldots$ such that

$$
N=\operatorname{dim} \bigoplus_{i=1}^{k} P\left(\left\{\lambda_{l}\right\}\right) B, \quad \operatorname{dim} P\left(\left\{\lambda_{n}\right\}\right) B=1, \forall n>N
$$

Let

$$
\left[x_{1}, \ldots, x_{N}\right]=\bigoplus_{i=1}^{k} P\left(\left\{\lambda_{l}\right\}\right) B, \quad\left[x_{n}\right]=P\left(\left\{\lambda_{n}\right\}\right) B, \forall n>N
$$

because

$$
P(\sigma(T))=I, \quad P\left(\left\{\lambda_{n} \mid n>N\right\}\right)=\mathrm{s}-\lim _{n} \bigoplus_{i=N+1}^{n} P\left(\left\{\lambda_{\sigma(i)}\right\}\right) B
$$

for every permutation $\sigma$ of $\{N+1, \ldots, n, \ldots\}$, so that $\left\{x_{n}\right\}_{n=1}^{\infty}$ is an unconditional basis of $B$. Therefore $T$ is $(D)$ type.

LEMMA 5. Let $\left\{x_{n}\right\}$ be an unconditional basis of Banach space $B$, $\left\|x_{n}\right\|=1, \forall n,\left\{y_{n}\right\}$ be a w-linearly independent sequence of $B$, i.e., if $\sum_{n} \alpha_{n} y_{n}=0$, then $\alpha_{n}=0, \forall n$. $B$

(1) If $\Sigma_{n}\left\|x_{n}-y_{n}\right\|<+\infty$, then $\left\{y_{n}\right\}$ is also an unconditional basis of

(2) If $B$ is a Hilbert space, and $\Sigma_{n}\left\|x_{n}-y_{n}\right\|^{2}<+\infty$, then $\left\{y_{n}\right\}$ is also an unconditional basis of $B$.

Proof. (1) It follows by [3], Ch. I, Th. 10.2, (a) $2^{0}$. $\Leftrightarrow 4^{0}$., and [3], Ch. II, Th. $17.1,1^{0}$. $\Leftrightarrow 2^{0}$;

(2) By [3], Ch. II, Th. 18.1, we can assume that $\left\{x_{n}\right\}$ is an orthogonal normalized basis of $B$. Let $N$ such that

$$
\sum_{n>N}\left\|x_{n}-y_{n}\right\|^{2}=\lambda^{2}<1, \quad 0 \leq \lambda<1
$$

and

$$
z_{n}=\left\{\begin{array}{rr}
x_{n} & 1 \leq n \leq N \\
y_{n} & n>N
\end{array}\right.
$$


then

$$
\sum_{n}\left\|x_{n}-z_{n}\right\|^{2}=\lambda^{2}<1
$$

Because of

$$
\left\|\sum_{n=1}^{m} \alpha_{n}\left(x_{n}-z_{n}\right)\right\|^{2} \leq \sum_{n=1}^{m}\left|\alpha_{n}\right|^{2} \sum_{n=1}^{m}\left\|x_{n}-z_{n}\right\|^{2} \leq \lambda^{2}\left\|\sum_{n=1}^{m} \alpha_{n} x_{n}\right\|^{2}
$$

for all finite sequences of numbers $\alpha_{1}, \ldots, \alpha_{m}$, so by [3], Ch. I, Th. 9.1, (b) $\delta$ ) and [3], Ch. II, Th. 17.1, $1^{0} . \Leftrightarrow 2^{0} .,\left\{z_{n}\right\}$ is also an unconditional basis of $B$.

Now by $\Sigma_{n}\left\|z_{n}-y_{n}\right\|<+\infty$, and 1) of this Lemma, $\left\{y_{n}\right\}$ is also an unconditional basis of $B$.

TheOREM 6. Let $T$ be a linear operator of $(D)$ type in Banach space $B,\left\{x_{n}\right\},\left\{\lambda_{n}\right\}$ and $N$ as in Definition $1,0 \in \rho(T)$. Let $V$ be a linear operator in $B$, such that $A=V T^{-\alpha}$ bounded, where $0 \leq \alpha<1$. Let $\nu_{n}=$ $\min _{m \neq n}\left|\lambda_{m}-\lambda_{n}\right|$ and we have one of the following conditions:

(1) $\Sigma_{n>N}\left(\left|\lambda_{n}\right|+\nu_{n}\right)^{\alpha} / \nu_{n}<+\infty$;

(2) If $B$ is a Hilbert space, and $\Sigma_{n>N}\left(\left|\lambda_{n}\right|+\nu_{n}\right)^{2 \alpha} / \nu_{n}^{2}<+\infty$;

(3) $\lim _{n}\left(\left|\lambda_{n}\right|+v_{n}\right)^{\alpha} / \nu_{n}=0$, and $\Sigma_{i, j}\left|a_{i j}\right|<\infty$, where $a_{i j}=f_{j}\left(A x_{i}\right)$, and $f_{j} \in B^{*}, f_{j}\left(x_{i}\right)=\delta_{\imath, j}$;

(4) $\left(\left|\lambda_{n}\right|+\nu_{n}\right)^{\alpha} / \nu_{n} \leq G, \forall n$, and $\Sigma_{i, j}\left|a_{i j}\right| \leq \beta$, where $a_{i j}$ as in (3), and $\beta$ is sufficiently small;

(5) If $B$ is a Hilbert space, $\left\langle x_{n}, x_{m}\right\rangle=\delta_{n, m}, \quad \forall n, m$, $\lim _{n}\left(\left|\lambda_{n}\right|+\nu_{n}\right)^{\alpha} / \nu_{n}=0$ and $A$ is a Hilbert-Schmidt operator;

(6) If $B$ is a Hilbert space, $\left\langle x_{n}, x_{m}\right\rangle=\delta_{n, m}, \forall n, m,\left(\left|\lambda_{n}\right|+\nu_{n}\right)^{\alpha} / \nu_{n} \leq$ $G, \forall n,\|A\|_{2} \leq \beta$, where \|\|$_{2}$ is Hilbert-Schmidt norm, and $\beta$ is sufficiently small, then $(T+V)$ is also $(D)$ type in $B$.

Proof. We can write $T=T_{s}+F$ such that $T_{s} x_{n}=\lambda_{n} x_{n}, n=1,2, \ldots$, and $F\left[x_{1}, \ldots, x_{N}\right] \subset\left[x_{1}, \ldots, x_{N}\right], F x_{n}=0, \forall n>N$. Using $(F+V)$ instead of $V$, we can assume that $T x_{n}=\lambda_{n} x_{n}, n=1,2, \ldots$ However, we can also assume $\left\|x_{n}\right\|=1, \forall n$. By [3], Ch. II, Th. 17.1, there exists a constant $M(\geq 1)$, such that

$$
\left\|\sum_{n} \beta_{n} \alpha_{n} x_{n}\right\| \leq M\|x\|, \quad \forall x=\sum_{n} \alpha_{n} x_{n} \in B \text { and }\left|\beta_{n}\right| \leq 1, \forall n .
$$

(1) Let $N_{1}$ sufficiently large $(>N)$ such that

$$
0<M \frac{a_{n}}{1-a_{n}}<1, \quad \forall n>N_{1}
$$

where $a_{n}=2 M\|A\|\left(\left|\lambda_{n}\right|+\nu_{n}\right)^{\alpha} / \nu_{n}$. 
For $n>N_{1}$, let $\Gamma_{n}$ be a circle whose center is at $\lambda_{n}$ and radius is $\nu_{n} / 2$. When $\lambda \in \Gamma_{n}$, because

$$
\left|\frac{\lambda_{m}^{\alpha}}{\lambda_{m}-\lambda}\right| \leq 2 \frac{\left(\left|\lambda_{n}\right|+\nu_{n}\right)^{\alpha}}{\nu_{n}} \quad \forall m
$$

so that $\|R(\lambda, T)\| \leq 2 M / \nu_{n},\|V R(\lambda, T)\| \leq a_{n}$. By [2], $(T+V)$ has one and only one single eigenvalue $\lambda_{n}(V)$ in $\Gamma_{n}$. Since $0<M a_{n} /\left(1-a_{n}\right)<1$, we can take

$$
x_{n}(V)=\sum_{l=0}^{\infty} \frac{-1}{2 \pi i} \int_{\Gamma_{n}}(-1)^{l} R(\lambda, T)[V R(\lambda, T)]^{l} d \lambda x_{n}
$$

as corresponding eigenvector. By

$$
\begin{aligned}
\left\|x_{n}(V)-x_{n}\right\| & \leq \sum_{l=1}^{\infty} \frac{1}{2 \pi} \int_{\Gamma_{n}}\|R(\lambda, T)\|\|V R(\lambda, T)\|^{l} d|\lambda| \leq M \sum_{l=1}^{\infty} a_{n}^{l} \\
& <4 M^{2}\|A\|\left(\left|\lambda_{n}\right|+\nu_{n}\right)^{\alpha} / \nu_{n}
\end{aligned}
$$

and condition 1 , so that

$$
\sum_{n>N_{1}}\left\|x_{n}(V)-x_{n}\right\|<+\infty
$$

However, since

$$
\begin{aligned}
&\left|\lambda_{n}(V)\right| \geq\left|\lambda_{n}\right|-\frac{\nu_{n}}{2} \geq\left|\lambda_{n}\right|-\frac{\left|\lambda_{n}-\lambda_{1}\right|}{2} \\
& \geq\left|\lambda_{n}\right|-\frac{1}{2}\left(\left|\lambda_{n}\right|+\left|\lambda_{1}\right|\right)=\frac{\left|\lambda_{n}\right|}{2}-\frac{\left|\lambda_{1}\right|}{2}
\end{aligned}
$$

so that $\lim _{n}\left|\lambda_{n}(V)\right|=+\infty$.

Let $\Gamma$ be a closed road, containing the points $\lambda_{1}, \ldots, \lambda_{N_{1}}$, and such that $\operatorname{dist}\left(\Gamma,\left\{\lambda_{1}, \ldots, \lambda_{N_{1}}\right\}\right)=\nu / 2$, where $\nu=\min _{n>N_{1}} \nu_{n}$. With the aid of [2], page 34 Lemma 4.10 and page 178 Theorem 6.17 we are able to show that $(T+V)$ has different eigenvalues $\lambda_{1}(V), \ldots, \lambda_{k}(V)\left(k \leq N_{1}\right)$ in $\Gamma$, and there exist linearly independent elements $x_{1}(V), \ldots, x_{N_{1}}(V)$ of $B$ such that

$$
(T+V)\left[x_{1}(V), \ldots, x_{N_{1}}(V)\right] \subset\left[x_{1}(V), \ldots, x_{N_{1}}(V)\right]
$$

and

$$
\sigma\left((T+V) \mid\left[x_{1}(V), \ldots, x_{N_{1}}(V)\right]\right)=\left\{\lambda_{1}(V), \ldots, \lambda_{k}(V)\right\} .
$$

Now it is sufficient to prove that $\left\{x_{n}(V) \mid n=1,2, \ldots\right\}$ is an unconditional basis of $B$. Because of Lemma 5 , we only need to prove that $\left\{x_{n}(V) \mid n=1,2, \ldots\right\}$ is $\omega$-linearly independent. 
Let

$$
\hat{P}_{n}=\frac{-1}{2 \pi i} \int_{\Gamma_{n}} R(\lambda, T+V) d \lambda \quad \forall n>N_{1}
$$

then

$$
\hat{P}_{n} x_{m}(V)=\delta_{n, m} x_{m}(V) \quad \forall m \in \mathbf{N} \text { and } n>N_{1} .
$$

If $\Sigma_{m} \beta_{m} x_{m}(V)=0$, then $0=\hat{P}_{n} \Sigma_{m} \beta_{m} x_{m}(V)=\beta_{n} x_{n}(V)$ and $\beta_{n}=0$, $\forall n>N_{1}$, and $\sum_{m=1}^{N_{1}} \beta_{m} x_{m}(V)=0$. But $\left\{x_{m}(V) \mid 1 \leq m \leq N_{1}\right\}$ is linearly independent, so that $\beta_{n}=0, \forall n$. This shows $\left\{x_{n}(V) \mid n=1,2, \ldots\right\}$ is $\omega$-linearly independent.

(2) Similarly to (1), let $N_{1}$ sufficiently large $(>N)$ such that

$$
0<M \frac{a_{n}}{1-a_{n}}<1 \quad \forall n>N_{1}
$$

where $a_{n}=2 M\|A\|\left(\left|\lambda_{n}\right|+\nu_{n}\right)^{\alpha} / \nu_{n}$.

For $n>N_{1}$, let $\Gamma_{n}$ as (1). When $\lambda \in \Gamma_{n}$, we also have $\|\mathrm{R}(\lambda, T)\| \leq$ $2 M / \nu_{n}$ and $\|V R(\lambda, T)\| \leq a_{n}$. Hence $(T+V)$ has one and only one single eigenvalue $\lambda_{n}(V)$ in $\Gamma_{n}$, and corresponding eigenvector is

$$
x_{n}(V)=\sum_{l=0}^{\infty} \frac{-1}{2 \pi i} \int_{\Gamma_{n}}(-1)^{l} R(\lambda, T)[V R(\lambda, T)]^{l} d \lambda x_{n} .
$$

It is obvious that $\lim _{n}\left|\lambda_{n}(V)\right|=+\infty$. We also take $\Gamma$ and $x_{1}(V), \ldots, x_{N_{1}}(V)$ as in (1). For $n>N_{1}$, as (1)

$$
\left\|x_{n}(V)-x_{n}\right\| \leq 4 M^{2}\|A\|\left(\left|\lambda_{n}\right|+\nu_{n}\right)^{\alpha} / \nu_{n} .
$$

By condition (2)

$$
\sum_{n}\left\|x_{n}(V)-x_{n}\right\|^{2}<+\infty
$$

Similarly as (1), $\left\{x_{n}(V) \mid n=1,2, \ldots\right\}$ is $\omega$-linearly independent. By Lemma $5,\left\{x_{n}(V) \mid n \in \mathbf{N}\right\}$ is an unconditional basis of $B$, so that $(T+V)$ is still $(D)$ type.

(3) Let

$$
\sup _{n}\left\|f_{n}\right\|=K, \quad b_{n}=\frac{1}{\nu_{n}} 2 M K \sum_{i j}\left|a_{i j}\right|\left(\left|\lambda_{n}\right|+\nu_{n}\right)^{\alpha} .
$$

Because of $\|A\|<K \Sigma_{i j}\left|a_{i j}\right|$, so $b_{n}>a_{n}$ (the definition of $a_{n}$, see (1)). By assumption, for large $n$,

$$
0<M \frac{b_{n}}{1-b_{n}}<1
$$


Let $\Gamma_{n}$ as (1), then there exists only one single eigenvalue $\lambda_{n}(V)$ of $(T+V)$ in $\Gamma_{n}$, and the corresponding eigenvector $x_{n}(V)$ has also the formula as (1). For $\lambda \in \Gamma_{n}$

$$
\begin{aligned}
& \left\|R(\lambda, T)[V R(\lambda, T)]^{l} x_{n}\right\| \\
& =\| \sum_{k_{1}} \cdots \sum_{k_{l}} \frac{\lambda_{n}^{\alpha}}{\lambda_{n}-\lambda} \frac{\lambda_{k_{1}}^{\alpha}}{\lambda_{k_{1}}-\lambda} \\
& \quad \cdots \frac{\lambda_{k_{l-1}}^{\alpha}}{\lambda_{k_{l-1}}-\lambda} \frac{\left\langle A x_{n}, f_{k_{1}}\right\rangle \cdots\left\langle A x_{k_{l-1}}, f_{k_{l}}\right\rangle}{\lambda_{k_{l}}-\lambda} x_{k_{l}} \| \\
& \leq\left[\frac{2\left(\left|\lambda_{n}\right|+\nu_{n}\right)^{\alpha}}{\nu_{n}}\right]^{l} \frac{2}{\nu_{n}} \sum_{k_{1}, \ldots, k_{l}}\left|a_{n k_{1}} \cdots a_{k_{l-1}} k_{l}\right| \\
& \leq\left[\frac{2\left(\left|\lambda_{n}\right|+\nu_{n}\right)^{\alpha}}{\nu_{n}}\right]^{l} \frac{2}{\nu_{n}}\left(\sum_{i j}\left|a_{i j}\right|\right)^{l-1} \sum_{k}\left|a_{n k}\right|
\end{aligned}
$$

so that

$$
\left\|x_{n}(V)-x_{n}\right\| \leq \sum_{l=1}^{\infty} b_{n}^{l} \sum_{k}\left|a_{n k}\right| / \sum_{i j}\left|a_{i j}\right|
$$

and $\Sigma_{n}\left\|x_{n}(V)-x_{n}\right\|<+\infty$. The rest part of proof is similar as (1).

(4) Take

$$
\beta<(2 M(M+1) K G)^{-1}
$$

the proof is similar as (3).

(5) Let

$$
c_{n}=2 M\|A\|_{2}\left(\left|\lambda_{n}\right|+\nu_{n}\right)^{\alpha} / \nu_{n}
$$

because $\|A\| \leq\|A\|_{2}$, so that $c_{n}>a_{n}$. If $n$ sufficiently large,

$$
0<M \frac{c_{n}}{1-c_{n}}<1
$$

Let $\Gamma_{n}$ as 1), then $\lambda_{n}(V), x_{n}(V)$ as (1). For $\lambda \in \Gamma_{n}$,

$$
\begin{aligned}
& \left\|R(\lambda, T)[V R(\lambda, T)]^{l} x_{n}\right\|^{2} \\
& \quad \leq\left[\frac{2\left(\left|\lambda_{n}\right|+\nu_{n}\right)^{\alpha}}{\nu_{n}}\right]^{2 l}\left(\frac{2}{\nu_{n}}\right)^{2}\|A\|_{2}^{2(l-1)} \sum_{k}\left|a_{n k}\right|^{2}
\end{aligned}
$$


and

$$
\left\|x_{n}(V)-x_{n}\right\|<\sum_{l=1}^{\infty} c_{n}^{l} \frac{1}{\|A\|_{2}}\left(\sum_{k}\left|a_{n k}\right|^{2}\right)^{1 / 2}
$$

so that

$$
\sum_{n}\left\|x_{n}(V)-x_{n}\right\|^{2}<+\infty
$$

The rest part of proof is similar as (2).

(6) Take

$$
\beta<(2 M(M+1) G)^{-1}
$$

the proof is similar as (5).

This completes the proof of Theorem 6 .

\section{REFERENCES}

1. N. Dunford and J. T. Schwartz, Linear Operators, Part III, New York, Wiley-Interscience, 1971.

2. T. Kato, Perturbation Theory for Linear Operators, Berlin, Springer, 1966.

3. I. Singer, Bases in Banach Spaces I, Springer-Verlag, 1970.

Received August 19, 1980 and in revised form May 11, 1981. Institute of Mathematics, Academia Sinica, Peking, China.

University of Pennsylvania

Philadelphia, PA 19104 


\section{PACIFIC JOURNAL OF MATHEMATICS \\ EDITORS}

DONALD BABBITT (Managing Editor)

University of California

Los Angeles, CA 90024

\section{Hugo Rossi}

University of Utah

Salt Lake City, UT 84112

C. C. Moore and Arthur Ogus

University of California

Berkeley, CA 94720
J. DugundiI

Department of Mathematics

University of Southern California

Los Angeles, CA 90089-1113

R. FINN and H. SAMELSON

Stanford University

Stanford, CA 94305

ASSOCIATE EDITORS
R. ARens
E. F. BECKENBACH
B. H. NeumanN
F. WOLF
K. YoshidA (1906-1982)

\section{SUPPORTING INSTITUTIONS}

UNIVERSITY OF ARIZONA

UNIVERSITY OF BRITISH COLUMBIA

CALIFORNIA INSTITUTE OF TECHNOLOGY

UNIVERSITY OF CALIFORNIA

MONTANA STATE UNIVERSITY

UNIVERSITY OF NEVADA, RENO

NEW MEXICO STATE UNIVERSITY

OREGON STATE UNIVERSITY
UNIVERSITY OF OREGON

UNIVERSITY OF SOUTHERN CALIFORNIA

STANFORD UNIVERSITY

UNIVERSITY OF HAWAII

UNIVERSITY OF TOKYO

UNIVERSITY OF UTAH

WASHINGTON STATE UNIVERSITY

UNIVERSITY OF WASHINGTON 


\section{Pacific Journal of Mathematics}

Vol. 104, No. 1

May, 1983

Nestor Edgardo Aguilera and Eleonor Ofelia Harboure de Aguilera, On

the search for weighted norm inequalities for the Fourier transform $\ldots \ldots .1$

Jin Akiyama, Frank Harary and Phillip Arthur Ostrand, A graph and its complement with specified properties. VI. Chromatic and achromatic numbers ......................................... 15

Bing Ren Li, The perturbation theory for linear operators of discrete type . . . 29

Peter Botta, Stephen J. Pierce and William E. Watkins, Linear

transformations that preserve the nilpotent matrices .............. 39

Frederick Ronald Cohen, Ralph Cohen, Nicholas J. Kuhn and Joseph

Alvin Neisendorfer, Bundles over configuration spaces .......... 47

Luther Bush Fuller, Trees and proto-metrizable spaces . . . . . . . . . . 55

Giovanni P. Galdi and Salvatore Rionero, On the best conditions on the

gradient of pressure for uniqueness of viscous flows in the whole space . . 77

John R. Graef, Limit circle type results for sublinear equations $\ldots \ldots \ldots \ldots 85$

Andrzej Granas, Ronald Bernard Guenther and John Walter Lee,

Topological transversality. II. Applications to the Neumann problem for

$y^{\prime \prime}=f\left(t, y, y^{\prime}\right) \ldots \ldots \ldots \ldots \ldots \ldots \ldots \ldots \ldots \ldots \ldots \ldots \ldots \ldots \ldots . \ldots 5$

Richard Howard Hudson and Kenneth S. Williams, Extensions of

theorems of Cunningham-Aigner and Hasse-Evans . . . . . . . . . . . 111

John Francis Kurtzke, Jr., Centralizers of irregular elements in reductive algebraic groups

James F. Lawrence, Lopsided sets and orthant-intersection by convex

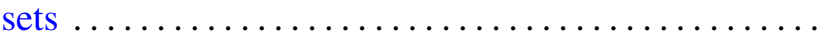

Åsvald Lima, G. H. Olsen and U. Uttersrud, Intersections of $M$-ideals and

$G$-spaces

Wallace Smith Martindale, III and C. Robert Miers, On the iterates of derivations of prime rings

Thomas H. Pate, Jr, A characterization of a Neuberger type iteration procedure that leads to solutions of classical boundary value problems

Carl L. Prather and Ken Shaw, Zeros of successive iterates of multiplier-sequence operators

Billy E. Rhoades, The fine spectra for weighted mean operators

Rudolf J. Taschner, A general version of van der Corput's difference theorem

Johannes A. Van Casteren, Operators similar to unitary or selfadjoint ones 